\title{
Contribution of a Wide-Swath Altimeter in a Shelf Seas Assimilation System: Impact of the Satellite Roll Errors
}

\author{
Matthieu Le Hénaff and Pierre De Mey \\ LEGOS, Université de Toulouse/CNRS, Toulouse, France \\ BAPTISTE MOURRE \\ Institut de Ciències del Mar, CSIC, Passeig Marítim, Barcelona, Spain \\ Pierre-Yves Le Traon \\ IFREMER Centre de Brest, Plouzané, France
}

(Manuscript received 24 May 2007, in final form 28 February 2008)

\begin{abstract}
The authors investigate the potential qualitative improvement brought by wide-swath, interferometrybased ocean altimetry measurements with respect to classical nadir altimeters in a coastal/shelf data assimilation system. In addition, particular attention is paid to roll errors, which could significantly reduce the expected benefits of wide-swath altimetry. A barotropic, nonlinear free-surface model is set up over the European shelf as part of an ensemble Kalman filter. Experiments assimilating simulated data are performed over the North Sea to test the ability of altimeter configurations to reduce model errors due to the action of meteorological forcing in the presence of bathymetric uncertainties. A simplified wide-swath observation scheme is used, composed of nadir altimeter height plus a nadir-centered cross-track sea level slope measurement. The simplified wide-swath measurements are found to be able to constrain events unsampled by a single nadir altimeter owing to a wider domain of influence in the cross-track direction and the ability to detect cross-track gradients.

Since the satellite-borne interferometer is highly sensitive to the platform behavior, especially satellite roll, experiments taking roll errors into account are then carried out. Whereas observational errors are considered independent in most data assimilation studies, the roll of the platform correlates those errors along the path of the satellite. Despite the large amplitude of the roll errors, the contribution of the wide-swath altimeter in coastal zones remains valuable as long as the roll frequency is known (within Gaussian error) and the assimilation scheme is designed to take observational error correlations into account.
\end{abstract}

\section{Introduction}

Altimeters have been routinely used for 15 years to measure sea level variability over the oceans. During that period, altimetric products have reached a high level of precision, allowing great progress in the understanding of the large-scale ocean circulation (Fu and Chelton 2001), the ocean mesoscale activity (Le Traon and Morrow 2001), sea level rise (Lombard et al. 2005), and the ocean tides (Le Provost 2001). Current altimetric missions have been designed to operate over the

Corresponding author address: Matthieu Le Hénaff, LEGOS, 14 Avenue Edouard Belin, 31400 Toulouse, France.

E-mail: matthieu.le.henaff@legos.obs-mip.fr open ocean and have significant shortcomings in coastal regions. These limitations include inadequate space and time sampling, contamination of altimeter and radiometer waveforms by land, and the inaccuracy of geophysical corrections. These questions are currently addressed, in particular through improved data sampling rate (Anzenhofer et al. 1999; Bouffard 2007) and by using specific algorithms and quality control procedures to improve corrections in those areas (Vignudelli et al. 2005). A wide-swath altimeter instrument based on radar interferometry appears very promising to improve the observation of the coastal ocean as it is theoretically able to measure sea level on a wide swath instead of only at the nadir of the satellite [Wide Swath Ocean Altimetry (WSOA) Fu 2003]. Despite the cancellation of the WSOA mission, such an instrument remains a 
serious candidate for future altimetric missions. A new project, called Surface Water Ocean Topography (SWOT), is presently under discussion to study both terrestrial surface waters and the coastal ocean. A description of the mission can be found in Alsdorf et al. (2007).

Using stochastic modeling, Mourre et al. (2004, hereafter MD04) studied the errors of a barotropic model of the European shelf due to meteorological forcing in the presence of uncertainties in bathymetry. Using that framework, Mourre et al. (2006, hereafter MD06) estimated the impact of various satellite configurations on the constraint of the model error on the North Sea shelf and the added impact of tide gauge measurements. The relative performances of the various simulated observation networks were evaluated by a statistical criterion based on an ensemble Kalman filter (EnKF) (Evensen 1994, 2003). The authors showed that tide gauges helped to constrain the short-scale model sea level error close to the coast. Farther away on the shelf, the dense spatial coverage of the simulated wide-swath altimetry measurements provides the adequate temporal sampling currently lacking in altimetric observations.

MD06 simulates interferometer observations by a high cross-track resolution within the swath $(15 \mathrm{~km})$ and a coarse error budget that ignores error correlations. In this paper, we adopt the modeling framework of MD06, with the objective of assessing, on the one hand, the first-order potential of wide-swath altimetry to detect coastal oceanic features by adding cross-track slope measurements to nadir observations and, on the other hand, the impact of the roll-induced along-track error correlations on the assimilation.

Indeed, the configuration used in MD06 does not provide a direct visualization of the first-order improvement expected from cross-track measurements compared to mere nadir measurements, namely, through monitoring the sea level slopes directly linked to currents at geostrophic scales, in both along and crosstrack directions. Moreover, their interferometer error budget did not take into account errors due to the behavior of the platform, especially satellite roll, which might reduce the expected benefits of wide-swath altimetry. Although it hardly affects a traditional radar altimeter as it just shifts the radar nadir footprint by a few meters, the satellite roll-induced displacement of an interferometer leads to large errors at the edges of the swath. Enjolras et al. (2006) have shown that error measurements for the WSOA mission on Jason-2 may reach an overall error budget of about $7 \mathrm{~cm}$ at the edge of the swath, with a large part due to the roll in the cross-track direction. From the unique standpoint of this type of variance-based argument, the amplitude of such errors would appear to be too large to fit the specifications for coastal applications ( $\mathrm{Fu}$ and Rodriguez 2004), thus raising concern about the feasibility of this type of mission. However, roll errors are special because they are correlated along the satellite path and their frequency, or frequencies, can be preestimated.

The scope of our results will be largely determined by the choice of the particular MD06 configuration. The model is barotropic and mainly reproduces the highfrequency response to atmospheric forcing. The modeling domain covers the whole western European shelves. The assimilation, using an EnKF, is reduced to the North Sea shelf. Although a wide swath altimeter observes several pixels in the cross-track direction, we use here a simplified interferometer observation model made of a nadir measurement and a cross-track slope measurement. The objectives of this simplification are 1) to determine the first order impact of cross-track measurements on sea level slopes associated with the model dynamics and 2) to readily account for crosstrack slope errors. Our focus is on the constraint of model errors on the part of the shelf that is farthest from the coast, where error scales are expected to be the most compatible with the sampling of a single wide swath altimeter. The model errors considered are the same as in MD04 and MD06, originating from the ocean response to meteorological forcing in the presence of bathymetric uncertainties, one of the major sources of errors on shelves as described in MD04.

The configuration of the barotropic ocean model, the main characteristics of the model error subspace, and the data assimilation scheme are briefly recalled in section 2, together with a description of the specific observation system used in our study. Section 3 describes the altimeter error budget. The results concerning the performance of wide swath measurements and the impact of the correlation of roll errors are presented in section 4. Conclusions are addressed in section 5.

\section{Impact assessment configuration}

\section{a. The model}

As in MD04 and MD06, we use the MOG2D model, developed by F. Lyard and D. Greenberg (2004, personal communication), and derived from Lynch and Gray (1979). It is a barotropic free-surface model solving the continuity and momentum shallow water equations on a finite element mesh through a single nonlinear wave equation. The solutions of these equations are in terms of sea level and barotropic zonal and meridional currents. The finite element mesh allows a fine 
resolution on steeper and shallower bathymetry. This model was designed to study the barotropic effects of tides, but it has been demonstrated to be adequate also to study the barotropic ocean response to high-freuency meteorological forcing (Carrère and Lyard 2003).

We use the MOG2D model with the same configuration and study period as MD04 and MD06. The model is implemented over the entire European shelf, from the North Sea to the Strait of Gibraltar. The focus is on the barotropic response of the North Sea to atmospheric forcing. Tidal forcing is not included (except in the form of tidal bottom drag). The simulations are forced by the European Centre for Medium-Range Weather Forecasts (ECMWF) winds and pressure, with a spatial resolution of $0.5^{\circ}$ and time step of $6 \mathrm{~h}$. The boundary conditions are based on the method of characteristics by Lardner et al. (1986), with no imposed inflow or outflow, since the particular ocean physics under study is less sensitive to the open ocean features. The approach allows gravity waves to leave the domain. The model is run from 4 to 31 December 1998, with a 2-day spinup period. During the study period, several meteorological events affect the modeling area. A low pressure system formed over Brittany around 15 December, associated with northerly winds over the North Sea. On 27 December, an intense atmospheric low crossed north of the North Sea. These events had a lifetime ranging from a few hours to $36 \mathrm{~h}$.

\section{b. Stochastic modeling and ensemble spread statistics}

As in MD04 and MD06, we are interested in constraining the model in the particular case of errors due to uncertainties in bathymetry. MD04 highlighted significant differences among the various bathymetric solutions available in the modeling domain. The main discrepancies are located in the very shallow areas along the coast and over the shelf break. They can lead to differences of more than $50 \%$ in terms of phase speed estimates $\left[(\mathrm{gH})^{-1 / 2}\right]$ of gravity waves, such as Kelvin waves which are known to travel along those coasts.

To characterize bathymetry errors and their impact in the model, MD04 set up a library of typical bathymetric differences by comparing five different databases. A new dataset of $O(100)$ bathymetry samples was then generated by a random combination of these typical errors added to the reference bathymetry from Service Hydrographique et Océanographique de la Marine (SHOM). Following the methodology of stochastic modeling, the model is run forced by the same atmo- spheric product with each bathymetry sample, providing an ensemble evolving in time. The model error is then approximated by the ensemble spread.

MD04 showed that the resulting errors were nonuniform over the domain. They are on average very low in the open ocean, where the bathymetry has little quantitative influence on surface elevation because of the thickness of the water column. The errors are larger in shelf seas, such as the English Channel or the North Sea. In these regions, waves are generated via the atmospheric forcing and propagate along the coasts. The physical characteristics of these waves and the generation process are highly sensitive to bathymetry. Overall, the bathymetric uncertainties studied here were also found to lead to higher model errors along the coasts than on the inner shelf. A notable exception is nevertheless found at the northern edge of the North Sea shelf. At that location, characterized by strong zonal winds and a particularly deep low pressure event during the study period, the model is shown to be sensitive to bathymetric perturbations applied at the shelf edge. A full description of the model error subspace in that context can be found in MD04.

This first ensemble of nonassimilated runs constitutes a reference ensemble to which the ensembles of simulations with data assimilation will be compared later in this paper (see experiment REF in Table 1).

\section{c. The assimilation system}

From the MD04 ensemble study, an ensemble Kalman filter has been set up, as in MD06. The EnKF is an approximation of the extended Kalman filter (EKF), which is itself an extension of the original Kalman filter to nonlinear processes. The EnKF is able to deal with nonlinear errors such as the ones in which we are interested. It is fully described in Evensen (2003) and briefly recalled here. The model forecast error covariance matrix $\mathbf{P}^{f}$ is given by the ensemble covariance matrix

$$
\mathbf{P}^{f}=E\left[\boldsymbol{\varepsilon}^{f} \boldsymbol{\varepsilon}^{f \mathbf{T}}\right] \approx \overline{\left(\mathbf{x}_{i}^{f}-\overline{\mathbf{x}_{i}^{f}}\right)\left(\mathbf{x}_{i}^{f}-\overline{\mathbf{x}_{i}^{f}}\right)^{\mathbf{T}}},
$$

where $E$ denotes mathematical expectation and the overbar the mean over the ensemble; $\mathbf{P}^{f}$ is approximated from the ensemble of simulations at each forecast time, $\boldsymbol{\varepsilon}^{f}$ is the model forecast error, and $\mathbf{x}_{i}^{f}$ is the model forecast state for each member $i$. In our experiments, the state vector is composed of the ocean variables, surface elevation, and barotropic zonal and meridional currents. Notice that the bathymetry is not corrected.

For each member $i$, the analyzed model state $\mathbf{x}_{i}^{\mathrm{a}}$ is 
TABLE 1. Description of the different configurations used for the data assimilation experiments.

\begin{tabular}{|c|c|c|c|c|}
\hline \multirow[b]{2}{*}{ Expt } & \multicolumn{3}{|c|}{ Parameters for the innovation vector generation } & \multirow[b]{2}{*}{ Comments } \\
\hline & $\begin{array}{c}\text { Nadir noise } \\
\text { error (sea level) }\end{array}$ & $\begin{array}{c}\text { Interferometer } \\
\text { noise error (XTS) }\end{array}$ & $\begin{array}{l}\text { Interferometer roll } \\
\text { uncertainty (std dev) }\end{array}$ & \\
\hline Ref & & & & No assimilation \\
\hline A & $3.9 \mathrm{~cm} \mathrm{rms}$ & None & None & Nadir alone \\
\hline $\mathrm{B}$ & $3.9 \mathrm{~cm} \mathrm{rms}$ & $4 \mathrm{~cm} \mathrm{rms}$ & None & No roll considered \\
\hline $\mathrm{C}$ & $3.9 \mathrm{~cm} \mathrm{rms}$ & $4 \mathrm{~cm} \mathrm{rms}$ & $0.0025 \mathrm{~Hz}$ & \\
\hline $\mathrm{D}$ & $3.9 \mathrm{~cm} \mathrm{rms}$ & $4 \mathrm{~cm} \mathrm{rms}$ & $0.0025 \mathrm{~Hz}$ & $\begin{array}{l}\text { Correlation ignored in the matrix } \mathbf{R} \text {, } \\
\text { larger amplitude on the diagonal }\end{array}$ \\
\hline $\mathrm{E}$ & $3.9 \mathrm{~cm} \mathrm{rms}$ & $4 \mathrm{~cm} \mathrm{rms}$ & $0.005 \mathrm{~Hz}$ & \\
\hline $\mathrm{F}$ & $3.9 \mathrm{~cm} \mathrm{rms}$ & $8 \mathrm{~cm} \mathrm{rms}$ & $0.0025 \mathrm{~Hz}$ & \\
\hline
\end{tabular}

calculated at any time when an observation or track is available-in our case, when the satellite flies over the domain, by adding to the forecast state $\mathbf{x}_{i}^{f}$ a linear combination of the individual corrections provided by the observations

$$
\mathbf{x}_{i}^{a}=\mathbf{x}_{i}^{f}+\mathbf{r b}_{i}
$$

with $\mathbf{r}$ the matrix of representers, calculated as

$$
\mathbf{r}=\mathbf{P}^{f} \mathbf{H}^{\mathrm{T}}
$$

and the vector $\mathbf{b}$ of representer coefficients being the solution of the system

$$
\left[\mathbf{H P}^{f} \mathbf{H}^{\mathrm{T}}+\mathbf{R}\right] \mathbf{b}_{i}=\mathbf{y}_{i}^{o}-\mathbf{H} \mathbf{x}_{i}^{f} \equiv \mathbf{d}:
$$

$\mathbf{d}$ is the so-called innovation vector, $\mathbf{H}$ is the projection matrix from the model space to the observation space, $\mathbf{R}$ is the observation error covariance matrix, $\mathbf{y}_{i}{ }^{o}$ is a simulated observation vector, and $\mathbf{H x}_{i}^{f}$ is the corresponding model proxy. The matrix of representers $\mathbf{r}$ describes the model error covariances between the model evaluated at observations locations and the model state variables. Each column of $\mathbf{r}$ can be used to explore the domain where the corresponding observation influences the solution, defining the domain of influence (DOI) of an observation by the area of significant correlation between the observed variable and the other state variables. Each simulated observation vector $\mathbf{y}_{i}^{o}$ is the sum of instantaneous measurements by the altimeter and of an observation error of covariance $\mathbf{R}$, which differs from one member to another, consistent with the EnKF methodology:

$$
\mathbf{y}_{i}^{o}=\mathbf{y}^{o}+\delta \mathbf{y}_{i}^{o} .
$$

In our study we consider two types of observations, so any observation vector has two components:

$$
\mathbf{y}_{i}^{o}=\left(\begin{array}{c}
\mathbf{y}_{i}^{n} \\
\mathbf{y}_{i}^{c}
\end{array}\right),
$$

with $\mathbf{y}_{i}^{n}$ containing the nadir sea level measurements and $\mathbf{y}_{i}^{c}$ containing the cross-track measurements by the interferometer. The reference observation $\mathbf{y}^{o}$ is calculated from one particular member that is later excluded from the ensemble, with no assimilation, called the control run. The way that the observation vectors are defined and the perturbations $\delta \mathbf{y}_{i}{ }^{o}$ are prescribed for both types of observations is explained in the following sections.

\section{d. The simplified measurement model}

The technical aspects of the wide swath altimeter in the WSOA configuration have been described by $\mathrm{Fu}$ (2003). More details have been given by Enjolras et al. (2006). A wide swath altimeter is composed of a nadir altimeter plus an interferometer. This latter instrument can be seen as adding sea level measurement points in the cross-track direction to the classical nadir altimeter data, or at first order as adding a cross-track slope measurement. We chose in this study to model the interferometer observations as cross-track slope for three reasons. First, the lower number of observations reduces the computational cost of the data assimilation, which is high when dealing with a large number of members in the ensemble since the inversion step in Eq. (4) is in observation space and is needed for each member. Second, such a choice allows a more qualitative analysis of the first-order impact of cross-track measurements on the model error control. Third, the specification of cross-track slope errors, such as the one (roll) considered in this paper, is more naturally achieved. Therefore, our study clearly cannot claim to quantify the impact of a wide swath altimeter; instead, we aim at understanding what the first-order impact of such an instrument is in our assimilation configuration and at describing the differential impact of design choices such as the platform roll error. In the following, we use a simplified model of a wide swath altimeter measuring both nadir sea level and the sea surface cross-track 


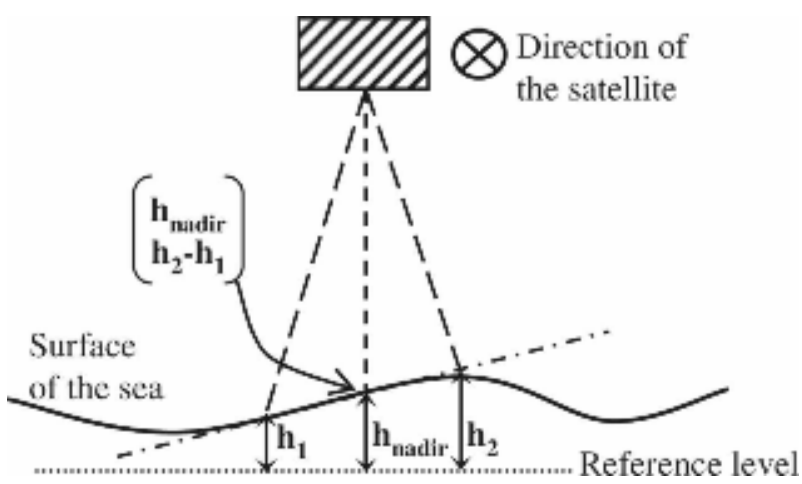

FIG. 1. Schematic representation of the wide-swath altimeter model with the two types of measurements: nadir sea level and sea level difference centered on nadir.

slope centered on the nadir. The latter is calculated from the difference in sea level between the two points $60 \mathrm{~km}$ away from the nadir on each side of the satellite in the cross-track direction, that is, the two points situated halfway within each half swath (Fig. 1). Hereafter, this measurement will be referred to as the cross-track slope (XTS), expressed in centimeters over the $120-\mathrm{km}$ half-swath centered on the nadir. Despite the loss of information due to the reduction of all cross-track pixel measurements into a single observation, this configuration is considered suitable for the study of barotropic flow in the North Sea. Indeed, MD04 showed that the DOI of a single sea level measurement in the North Sea farther from the coast is about $220 \mathrm{~km}$ wide, corresponding to a correlation of 0.6 (MD04, Fig. 14). This is an estimate of a typical space scale for model sea level error structures in the central North Sea. The geometric basis of $120 \mathrm{~km}$ for the XTS measurement would therefore allow one to sample such events in the error subspace. The along-track spatial resolution of the measurements is $15 \mathrm{~km}$. Our model satellite flies on the same orbit as Jason-2 with a 10-day period (see Fig. 2).

As part of real data processing, the correction of the sea level measurements errors due to the wet troposphere is usually calculated from measurements at the nadir of the satellite. Thus, when the nadir reaches the continent, those corrections are not valid anymore, and the whole swath has to be discarded unless data are specially processed to extrapolate the nadir correction. In our study, for the sake of realism, the whole swath has been discarded in such cases. Altimetric mission designers are currently working on a way to overcome this difficulty. To that end, alternative methods have been used in the treatment of continental hydrological data, using in situ measurements and ground models (V. Enjolras 2004, personal communication), and an

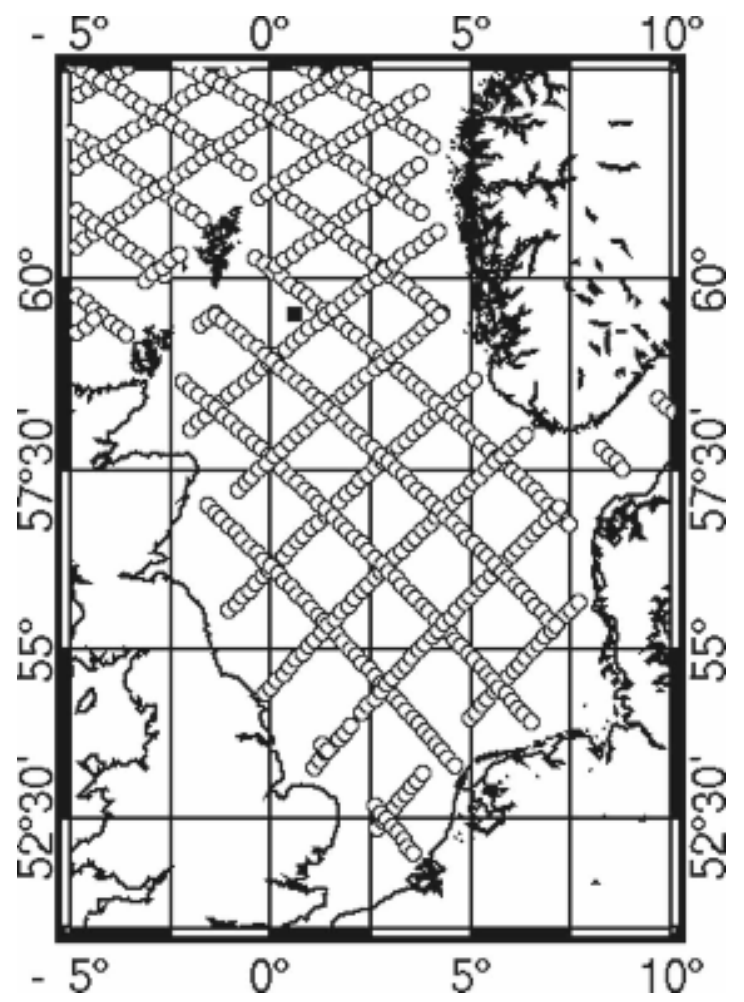

FIG. 2. Tracks of the simulated wide swath altimeter over the study domain. The black square is point 1 .

adapted data processing in the land-sea transition zone by Desportes et al. (2007) has given promising results.

\section{Characterization and modeling of the wide-swath altimeter errors}

In this section, we describe the different sources of errors of the instrument and their technical aspects. This will allow us to specify the error terms added to reference observation $\mathbf{y}^{o}$ in the ensemble Kalman filter formalism, that is, the $\delta \mathbf{y}_{i}^{o}$ vectors, each composed of a nadir sea level error vector $\delta \mathbf{y}_{i}^{n}$ and a cross-track slope error vector $\delta \mathbf{y}_{i}^{s}$ :

$$
\delta \mathbf{y}_{i}^{o}=\left(\begin{array}{c}
\delta \mathbf{y}_{i}^{n} \\
\delta \mathbf{y}_{i}^{s}
\end{array}\right) .
$$

\section{a. Errors of the nadir altimeter}

Consistent with previous work cited above, our nadir altimeter is considered to have an instrumental rms error of $3.9 \mathrm{~cm}$, which is larger than the $2-\mathrm{cm}$ rms standard error commonly adopted in the open ocean $(\mathrm{Fu}$ 2003; Le Traon and Ogor 1998). Larger errors in alti- 
metric data in coastal zones are attributable to several sources. The correction of the wet troposphere effects is perturbed by the presence of the coast (Desportes et al. 2007). Access to altimetric data close to the coast thus requires a special processing called retracking (Anzenhofer et al. 1999), based on different assumptions than in the open ocean, where the focus is instead on basin scales (Le Traon and Ogor 1998). The model-based correction of tides and high frequency response to meteorological forcing also introduce larger uncertainties as these processes are of much larger amplitude on the continental shelf (Andersen 1999). The value of $3.9 \mathrm{~cm}$ for nadir sea level observation error is considered realistic in our study where tides are not present: it is very close to values of the error budget found for new processed data from current altimeters of the coastal zone in the northwestern Mediterranean Sea where tides are of very low amplitude (Bouffard 2007).

Note that nadir altimeters are almost insensitive to platform roll: the radar footprint on the ground would just be translated by a few meters, hardly modifying the distance between the footprint and the satellite, thus leading to a negligible error in the sea level measurement. The nadir altimeter observation errors are thus uncorrelated, at least through platform roll.

The $\delta \mathbf{y}_{i}^{n}$ vector used in the assimilation step is randomly generated using a Gaussian distribution with 0 mean and 3.9- $\mathrm{cm}$ standard deviation. The part of the observation error matrix $\mathbf{R}$ dedicated to the nadir sea level measurement is then purely diagonal with homogeneous variance $(3.9 \mathrm{~cm})^{2}$.

\section{b. Errors of the interferometer}

Here, in contrast to previous studies, we consider the interferometer-related errors to be composed of two sources of errors: an instrumental error and an error due to roll of the satellite:

$$
\delta \mathbf{y}_{i}^{s}=\delta \mathbf{y}_{i}^{\text {instr }}+\delta \mathbf{y}_{i}^{\text {roll }}
$$

We chose the instrumental error $\delta \mathbf{y}_{i}^{\text {instr }}$ to be $4 \mathrm{~cm} /$ $120 \mathrm{~km} \mathrm{rms}$ in XTS. Enjolras et al. (2006) actually showed that the corresponding error in the XTS calculated from instrumental errors at the middle of the swath reaches $6.3-\mathrm{cm} \mathrm{rms}$. This lower value for the instrumental error in our study attempts to compensate for the lack of density of cross-track measurements due to the simplified scheme that we chose. Like any working hypothesis, this is questionable, but we feel that the main qualitative results of this paper do not critically depend on the detailed instrumental error budget adopted.

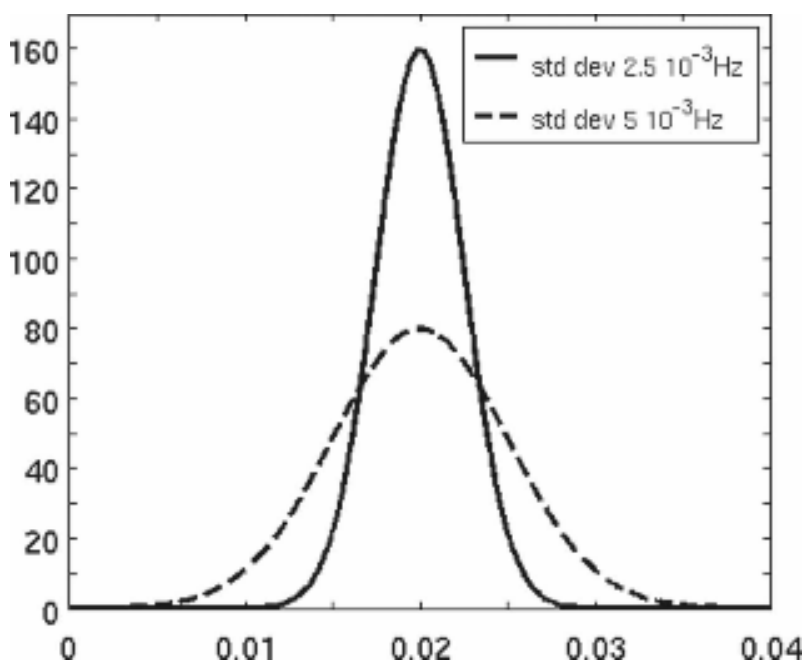

FIG. 3. Distribution of the roll error frequency around the mean value of $0.02 \mathrm{~Hz}$ for two study cases $\left(10^{4}\right.$ elements for the test).

The error due to the platform roll $\delta \mathbf{y}_{i}^{\text {roll }}$ is an additional XTS error corresponding to a slope error of amplitude 0.2 arcsec. This value is larger than the largest uncertainty on the roll evaluation considered in the study by Enjolras et al. (2006). Indeed, in the coastal ocean the crossover maximum likelihood technique required to reduce the roll angle during the ground postprocessing may not be as efficient as in the open ocean. An amplitude of 0.2 arcsec corresponds to a XTS measurement of $11.6 \mathrm{~cm}$ over the $120-\mathrm{km}$ measurement basis, which is much larger than the instrumental error. The errors due to roll are correlated along the path of the satellite, with a dominant roll frequency close to $0.02 \mathrm{~Hz}$ for the Jason-2 platform initially chosen to host the WSOA instrument. This is a low enough frequency to be well modeled by the on-board and ground processing (V. Enjolras 2006, personal communication). This frequency corresponds to an along-track wavelength of $350 \mathrm{~km}$. An uncertainty has been subsequently added to the central frequency to yield an along-track decreasing correlation: a Gaussian distribution of $0 \mathrm{~Hz}$ mean and $0.0025 \mathrm{~Hz}$ standard deviation has been used to randomly generate error samples added to the central frequency. A specific case illustrates the role of the uncertainty in frequency by using a $0.005 \mathrm{~Hz}$ standard deviation instead of $0.0025 \mathrm{~Hz}$. The distributions of frequency in the two cases are shown in Fig. 3. This along-track correlation implies that the nondiagonal elements of the matrix R corresponding to the XTS measurements are now nonzero.

Together, the XTS errors due to the noise and to the roll make up the $\delta \mathbf{y}_{i}^{s}$ terms used (1) to perturb the observations and (2) to approximate the matrix $\mathbf{R}$ offline 
as the statistical covariance matrix on a large ensemble (10 $0^{4}$ members):

$$
\begin{aligned}
\mathbf{R} & =E\left[\delta \mathbf{y}^{o} \delta \mathbf{y}^{o \mathrm{~T}}\right] \\
& =E\left[\left(\begin{array}{c}
\delta \mathbf{y}^{n} \\
\delta \mathbf{y}^{\mathrm{s}}
\end{array}\right)\left(\begin{array}{c}
\delta \mathbf{y}^{n} \\
\delta \mathbf{y}^{s}
\end{array}\right)^{\mathrm{T}}\right] \\
& =E\left[\left(\begin{array}{c}
\delta \mathbf{y}^{n} \\
\delta \mathbf{y}^{\text {instr }}+\delta \mathbf{y}^{\text {roll }}
\end{array}\right)\left(\begin{array}{c}
\delta \mathbf{y}^{n} \\
\delta \mathbf{y}^{\text {instr }^{\text {roll }}}+\delta \mathbf{y}^{\text {rol }}
\end{array}\right)^{\mathrm{T}}\right] .
\end{aligned}
$$

Figure 4 shows the full $\mathbf{R}$ matrix. The part of $\mathbf{R}$ dedicated to the interferometer error budget is composed of a full matrix block attributable to the roll correlation plus a homogeneous diagonal block due to the instrumental noise $\delta \mathbf{y}_{i}^{\text {instr }}$. The interferometer and nadir errors are assumed to be uncorrelated.

\section{Impact of cross-track measurements}

The impact of the wide-swath altimeter model in our assimilation system is analyzed through several experiments from spatial, temporal, and space-time statistics.

\section{a. The study configurations}

Table 1 describes the experiments carried out in the EnKF framework. Experiment A is nadir only, while experiment $\mathrm{B}$ is nadir plus interferometer with no roll of the platform. Together, experiments A and B show the impact of "idealistic" cross-track measurements and the physical aspects linked to this type of observation. Experiments $\mathrm{C}$ and $\mathrm{D}$ are nadir plus interferometer with the platform rolling, but $\mathrm{C}$ takes that roll into account in the $\mathbf{R}$ matrix whereas $\mathrm{D}$ ignores the corresponding along-track correlation. In both experiments the XTS observation error $\delta \mathbf{y}_{i}^{s}$ used to generate the innovation vector $\mathbf{d}$ is defined as in section $3 \mathrm{~b}$. Case $\mathrm{C}$ deals with the correlation of the errors in the data assimilation process where matrix $\mathbf{R}$ is modified to this end, as in section $3 b$. Case $\mathbf{D}$ assumes that the errors are uncorrelated during the assimilation step, the matrix $\mathbf{R}$ being purely diagonal with an amplitude equal to $(4+$ $11.6)^{2}$, that is, the square of the sum of the instrumental error and of the maximum roll-induced error. Experiments $\mathrm{E}$ and $\mathrm{F}$ are sensitivity tests with respect to Experiment $\mathrm{C}$ on the amplitude of the uncertainty on the satellite roll frequency (E) and on the interferometer instrumental error $(\mathrm{F})$.

\section{b. Spatial impact of XTS measurements}

Let us start with spatial impact as shown by experiment B. The differential impact of one XTS measure-

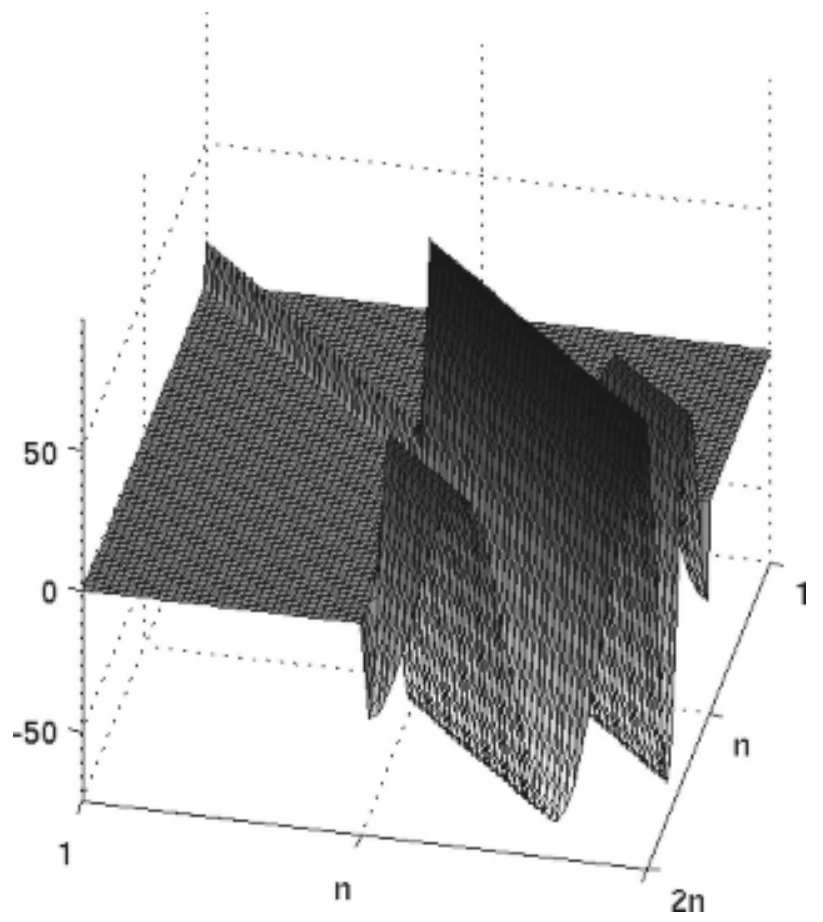

FIG. 4. Three-dimensional view of an observational covariance error matrix. The number of observation locations is $n=35$. Ranks within $[1, n]$ are for nadir sea level; ranks within $[n+1,2 n]$ are for XTS measurements.

ment can be illustrated by means of the matrix of representers [Eq. (3)] that contains the error-space covariances between an observation and the model state variables. In particular, we evaluate the spatial impact of an observation by the interferometer by comparing the DOIs of both types of measurements, nadir and XTS. Figure 5 shows the average, over all the measurements from the satellite track, of the normalized representer [columns of matrix $\mathbf{r}$ in Eq. (3)], giving access to an average DOI for each measurement type along the full track. Significant correlation for the average DOIs is considered 0.2 or higher. On Fig. 5, the average DOI in sea level for the nadir measurement is restricted to the vicinity of the path of the satellite, whereas the XTS measurement average DOI is more spread in the cross-track direction. The slope information provided by the XTS measurement appears in the form of negative and positive sea level lobes on each side of the satellite path. Moreover, considering the southern part of the track, the XTS measurement has its largest influence in an area that is totally unsampled by the nadir altimeter, along the German and Dutch coasts. This illustrates the fact that the interferometer is theoretically able to bring new information and to extend the range of a classical altimeter by means of its slope measurement (at first order). 


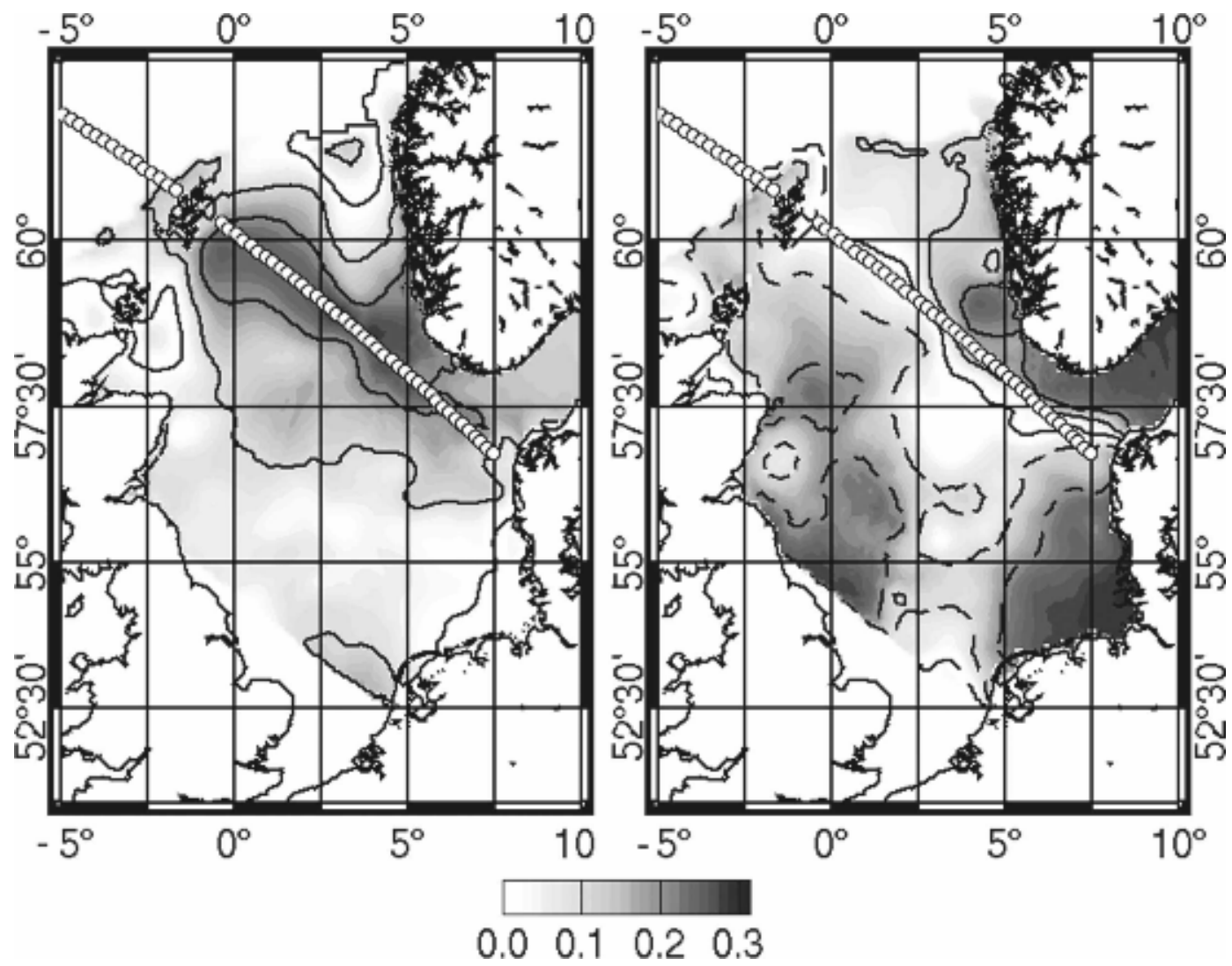

FIG. 5. Average of correlations between model sea level and (left) along-track nadir sea level and (right) XTS measurements for all the points of the plotted track for experiment B at 1000 UTC 18 Dec. Continuous lines are for positive values; dashed lines are for negative values. The color bar indicates the amplitude.

\section{c. Temporal impact}

The performance of the various observational system options considered above is now evaluated in terms of model error variance reduction, approximated in the EnKF by ensemble spread reduction between forecast and analysis, as in MD04 and MD06. Figure 6 compares the temporal evolutions of the sea level ensemble variances at a specific point of the North Sea (point 1 in Fig. 2 ) by a single nadir altimeter (experiment $A$ ) and by the full nadir plus interferometer system without roll error (experiment B). These are compared to the evolution of the ensemble variances of the reference ensemble (experiment REF) to quantify the ensemble variance reduction in the two experiments. Mean ensemble variance reduction increases from $19 \%$ in experiment $\mathrm{A}$ to more than $26 \%$ in experiment B owing to the crosstrack slope measurement. Although this increase is significant, we believe that the added value of the interferometer would be further enhanced if 1) all swath pixels were used and 2) the bathymetry was corrected by assimilation, leading to better velocity forecasts. Moreover, as pointed out in MD06, the improvements brought by the interferometer are not stationary over the period, but happen at specific time steps when the cross-track measurement brings clear additional information compared to the nadir sea level. For instance, the local error variance rises at point 1 during storm events, namely around 17 and 28 December (Fig. 6); this is when the clearest contribution of the interferometer is seen since the instrument is able to detect the (erroneous) sea level slopes generated by atmospheric forcing in the presence of bathymetry errors.

\section{d. Global error reduction}

Various configurations are now compared in terms of global error variance reduction in the model variables, namely sea level and zonal and meridional velocities (Fig. 7). For any data assimilation experiment, we define the global error variance reduction as the percentage of reduction of ensemble variance, averaged over the study period and domain, with respect to the ensemble in which no data are assimilated. The global error reduction in terms of barotropic velocities is overall less efficient than the one in sea level: this differen- 


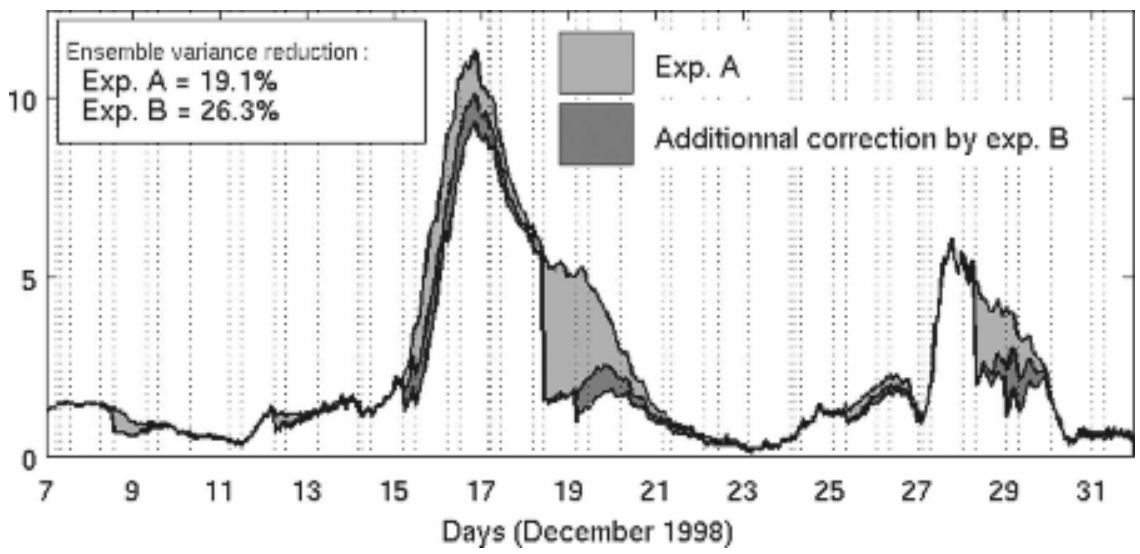

FIG. 6. Sea level ensemble variances $\left(\mathrm{cm}^{2}\right)$ at point 1 . The upper curve represents experiment REF, the middle one experiment $\mathrm{A}$, and the lower one experiment $\mathrm{B}$. The light gray area is the ensemble variance reduction relative to experiment $\mathrm{A}$, and the dark gray area is the additional reduction obtained in experiment $\mathrm{B}$.

tial behavior in error reduction has been discussed in MD06. They found two main reasons. First, as the correction of the velocities was made through a multivariate statistical relationship between the observed sea level and the model velocities, through the matrix $\mathbf{P}^{f}$, it was less efficient than it would have been through direct assimilation of velocities. Second, the bathymetry, which is the parameter affected by error in our configuration, strongly affects the velocity; since it is not corrected here, the errors in velocities stay high. Moreover, in our study, as the interferometer measurement is reduced to a single sea level difference between two points, the correction does not benefit from the high spatial resolution of the interferometer in the crosstrack direction, which would undoubtedly have an impact on the velocity correction because of the smaller length scale of the barotropic velocity error features. (These errors are located where the sea level gradients are maximum and, thus, have a shorter spatial extension than sea level errors.)

The addition of cross-track measurements in experiment B improves the control of the model, especially in terms of currents, with an added error reduction of about $50 \%$. Experiment $\mathrm{C}$, which now assumes correlated roll errors both in the simulated observations and in matrix $\mathbf{R}$, shows that, even if these errors are very

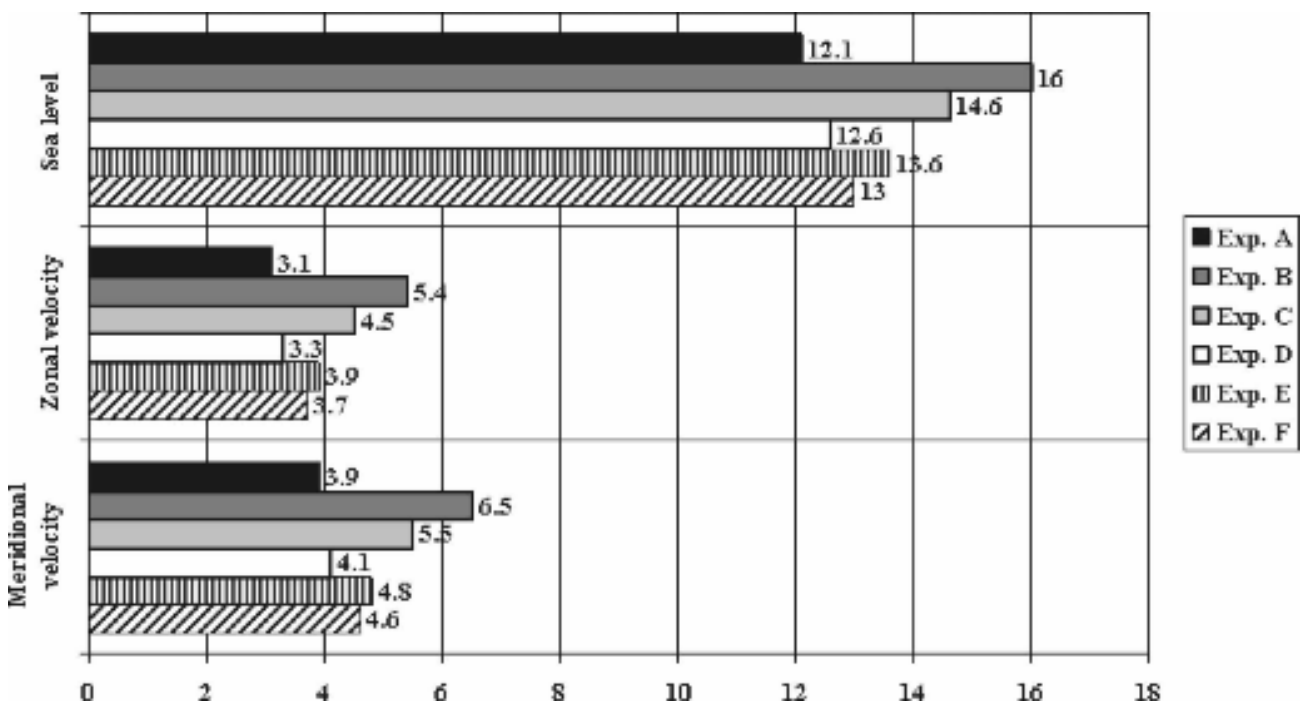

FIG. 7. Reduction of error variances in the North Sea, expressed as a percentage, for all experiments of the study: sea level, zonal, and meridional velocities. 
large in amplitude (more than $11 \mathrm{~cm}$ over $120 \mathrm{~km}$ in XTS compared to 4-cm instrumental error), the instrument remains useful compared to a classical nadir altimeter since the results are closer to those of experiment $\mathrm{B}$ than to experiment A. Experiment D supports that this performance is due to the adapted data assimilation scheme, which takes into account the alongtrack correlation of the roll errors: when the roll error, while present and correlated, is modeled as additional random noise in the assimilation process, then the cross-track measurement appears to be almost useless when compared to a single nadir altimeter.

Experiments $\mathrm{E}$ and $\mathrm{F}$ are sensitivity tests on both the uncertainty concerning the roll frequency and the instrumental noise of the interferometer. It appears that a higher uncertainty on the roll frequency (experiment E, second case in Fig. 3), with a frequency distribution spread between 0 and $0.04 \mathrm{~Hz}$, still gives better results than a single nadir altimeter. In contrast, an increase in the instrumental error of the interferometer (experiment $\mathrm{F}, 8 \mathrm{~cm}$ over $120 \mathrm{~km}$ instead of $4 \mathrm{~cm}$ ) leads to worse results than for experiment $\mathrm{E}$. We now attempt to examine the reasons behind those two results. Figure 8 shows the full spectra of matrix $\mathbf{R}$ in the experiments of our study: $n$ is the number of observation locations, so $2 n$ is the observation subspace dimension when we consider the two kinds of measurements, nadir plus cross-track slope. The eigenvalues between 1 and $n$ are associated with measurement errors in XTS, and the part of the spectra between $n+1$ and $2 n$ is associated with the error in nadir sea level measurements, characterized by smaller amplitude. Including correlated roll errors in the $\mathbf{R}$ matrix-as in experiment $\mathrm{C}, \mathrm{E}$, and $\mathrm{F}$-only modifies a few eigenvalues of about $n / 5$. The eigenvalues associated with the roll are of much higher amplitude than those associated with measurement noise. We now use the fact that $\mathbf{R}$ is a positive definite matrix, so the eigenvalues of its inverse are the inverse eigenvalues of $\mathbf{R}$, and the order of the eigenvectors is reversed. In the data assimilation process, the $\mathbf{R}$ matrix is involved in the calculation of the vector $\mathbf{b}$ of representer coefficients in Eq. (4), where $\mathbf{R}^{-1}$ roughly acts as a multiplying factor to the innovation vector $\mathbf{d}$ (obviously, the lesser the error, the stronger the constraint). The few dominating eigenvalues of $\mathbf{R}$, due to roll, are of very low amplitude in the spectrum of $\mathbf{R}^{-1}$, which is dominated instead by the eigenvalues from the measurement noise, in sea level and XTS. Dealing with higher uncertainties about the roll frequency (experiment E), thus only marginally affects this step of the data assimilation process, while increasing the measurement noise error (experiment F) has a more critical

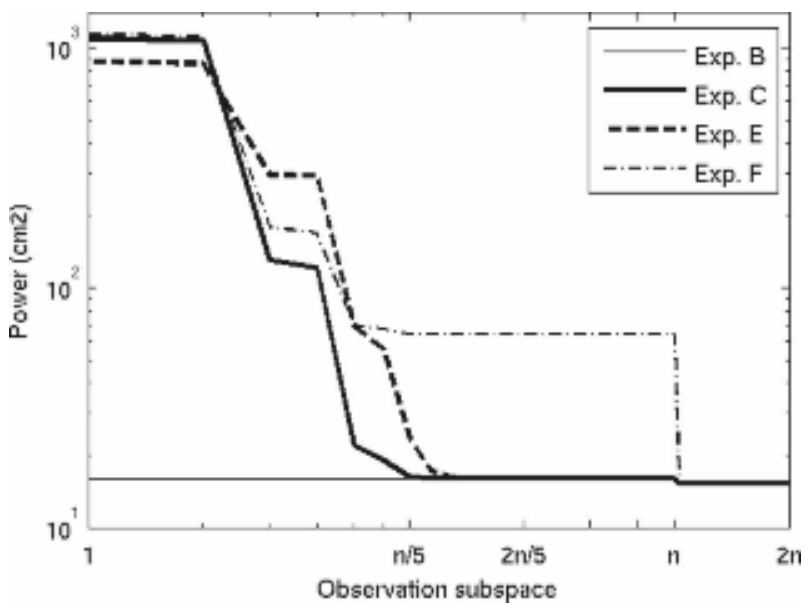

FIG. 8. Logarithmic representation of spectra of eigenvalues for study cases with various $\mathbf{R}$ matrices ( $n$ : number of track points; $2 n$ : total number of observations, including nadir sea level and XTS; here $n=35$ ).

effect since it affects a large number of dominant eigenvalues of $\mathbf{R}^{-1}$. Therefore, the uncertainty in the roll frequency distribution around its mean appears to be less important than the amplitude of the cross-track slope noise, once the roll is taken into account with correct central frequency.

\section{Conclusions}

We investigated the potential qualitative improvement brought by wide-swath, interferometry-based ocean altimetry measurements in a coastal/shelf data assimilation system with respect to classical nadir altimeters. Following the approach of MD06, a simplified wide-swath altimeter observing system model has been set up to study the impact of wide swath altimetry in reducing model errors due to the action of meteorological forcing in the presence of bathymetric uncertainties in the North Sea, using an ensemble Kalman filter. The study also investigates the impact of alongtrack correlated errors of high amplitude due to platform roll, which could significantly reduce the expected benefits of the wide-swath altimeter.

Cross-track measurements are of great interest since they allow one to sample sea level gradients in both along- and cross-track directions. These measurements, which are related to velocities in our system, exhibit wider domains of influence than nadir sea level. In addition, in contrast to nadir altimetry, the entire shelf domain is sampled, associated with shorter revisiting times. This allows for a better control of temporal changes in both sea level and velocities. The improvement is not constant in time: it is found to depend on 
the ocean state and the information provided by the interferometer overflights.

As expected, the roll of the satellite reduces the performance of the altimeter when compared to a rollerror-free system. But, despite the high amplitude of those roll errors, the wide-swath observation system remains valuable when compared to a single nadir, as long as the roll error is adequately taken into account in the data assimilation process, in particular with correct central frequency. The performance of the instrument is found to be more sensitive to instrumental noise of the interferometer than to the uncertainties associated with the roll frequency.

Results in this study depend on the modeling and data assimilation configuration chosen, namely the simplified interferometer model, the particular North Sea ocean dynamics, the error-space dynamics (bathymetry errors), and the duration of the experiments. These limitations have been discussed in the text. Nevertheless, the methodology presented in this paper has the potential to be used in more complex configurations. In particular, to get a more accurate assessment of the quantitative impact of the wide swath altimeter, a more realistic observation model would have to be substituted, using all available measurement points in the cross-track direction, as well as the associated realistic instrumental errors (e.g., as in Enjolras et al. 2006).

Similarly, even though this study relied on the particular WSOA mission on Jason-2, the orders of magnitude of roll error amplitude and frequency that we used are close to those specified by altimetric mission designers to achieve sufficient precision for observing sea level. As a matter of fact, whatever the actual roll behavior of the platform, the figures in this paper are typical of the residual roll errors after ground processing (V. Enjolras 2006, personal communication). Therefore, we are confident that our results constitute a valuable contribution in support of the design of future missions such as SWOT. We hope that this study can also provide some useful indications about the relative importance of the different terms of the error budget. Finally, the methodology used here stresses the power of ensemble-based methods to infer observationspace statistics and the need for mission designers to make their design choices based on state-of-the-art deterministic/stochastic modeling of the observed processes.

Acknowledgments. The authors gratefully thank Vivien Enjolras for his advice regarding the WSOA error budget and Nelly Mognard for her insights into the future of wide swath altimetry and the SWOT mission. Support of Matthieu Le Hénaff by Délégation
Générale pour l'Armement is acknowledged. We also thank two anonymous reviewers for very useful suggestions and comments.

\section{REFERENCE}

Alsdorf, D. E., E. Rodríguez, and D. P. Lettenmaier, 2007: Measuring surface water from space. Rev. Geophys., 45, RG2002, doi:10.1029/2006RG000197.

Andersen, O. B., 1999: Shallow water tides in the northwest European shelf region from TOPEX/POSEIDON altimetry. $J$. Geophys. Res., 104 (C4), 7729-7741.

Anzenhofer, M., C. K. Shum, and M. Rentsh, 1999: Coastal altimetry and applications. Ohio State University Geodetic Science and Surveying Tech. Rep. 464, 36 pp.

Bouffard, J., 2007: Amélioration de l'altimétrie côtière appliquée à l'étude de la circulation dans la partie nord du bassin occidental méditerranéeen. Ph.D. thesis, Université Paul Sabatier, $223 \mathrm{pp}$.

Carrère, L., and F. Lyard, 2003: Modeling the barotropic response of the global ocean to atmospheric wind and pressure forcing-Comparisons with observations. Geophys. Res. Lett., 30, 1275, doi:10.1029/2002GL016473.

Desportes, C., E. Obligis, and L. Eymard, 2007: On the wet tropospheric correction for altimetry in coastal regions. IEEE Trans. Geosci. Remote Sens., 45, 2139-2149.

Enjolras, V., P. Vincent, J. C. Souyris, E. Rodriguez, L. Phalippou, and A. Cazenave, 2006: Performances study of interferometric radar altimeters: From the instrument to the global mission definition. Sensors, 6, 164-192.

Evensen, G., 1994: Sequential data assimilation with a nonlinear quasi-geostrophic model using Monte Carlo methods to forecast error statistics. J. Geophys. Res., 99 (C5), 10 143-10 162. , 2003: The ensemble Kalman filter: Theoretical formulation and practical implementation. Ocean Dyn., 53, 343-367.

Fu, L.-L., Ed., 2003: Wide-swath altimetric measurement of ocean surface topography. Jet Propulsion Laboratory Publication 03-002, 67 pp.

—, and D. B. Chelton, 2001: Large-scale ocean circulation and variability. Satellite Altimetry and Earth Sciences: A Handbook of Techniques and Applications, L.-L. Fu and A. Cazenave, Eds., Academic Press, 133-169.

— , and E. Rodriguez, 2004: High-resolution measurement of ocean surface topography by radar interferometry for oceanographic and geophysical applications. State of the Planet: Frontiers and Challenges, Geophys. Monogr., Vol. 150, Amer. Geophys. Union, 209-224.

Lardner, R. W., H. M. Cekirge, and N. Gunay, 1986: Numerical solution of the two-dimensional tidal equations using the method of characteristics. Comput. Math. Appl., 10, 10651080.

Le Provost, C., 2001: Ocean tides. Satellite Altimetry and Earth Sciences: A Handbook of Techniques and Applications, L.-L. Fu and A. Cazenave, Eds., Academic Press, 267-303.

Le Traon, P.-Y., and F. Ogor, 1998: ERS-1/2 orbit improvement using TOPEX/POSEIDON: The $2 \mathrm{~cm}$ challenge. J. Geophys. Res., 103 (C4), 8045-8058.

- and R. Morrow, 2001: Ocean currents and mesoscale eddies. Satellite Altimetry and Earth Sciences: A Handbook of Techniques and Applications, L.-L. Fu and A. Cazenave, Eds., Academic Press, 171-215.

Lombard, A., A. Cazenave, P.-Y. Le Traon, and M. Ishii, 2005: 
Contribution of thermal expansion to present-day sea-level change revisited. Global Planet. Change, 47, 1-16.

Lynch, D. R., and W. G. Gray, 1979: A wave equation model for finite element tidal computation. Comput. Fluids, 7, 207-228.

Mourre, B., P. De Mey, F. Lyard, and C. Le Provost, 2004: Assimilation of sea level data over continental shelves: An ensemble method for the exploration of model errors due to uncertainties in bathymetry. Dyn. Atmos. Oceans, 38, 93-121, doi:10.1016/j.dynatmoce.2004.09.001.
Y. Ménard, F. Lyard, and C. Le Provost, 2006: Relative performance of future altimeter systems and tide gauges in constraining a model of North Sea high-frequency barotropic dynamics. Ocean Dyn., 56, 473-486.

Vignudelli, S., P. Cipollini, L. Roblou, F. Lyard, G. P. Gasparini, G. R. M. Manzella, and M. Astraldi, 2005: Improved satellite altimetry in coastal systems: Case study of the Corsica Channel (Mediterranean Sea). Geophys. Res. Lett., 32, L07608, doi:10.1029/2005GL022602. 